\section{DETFAC: A program for assessing factor indeterminacy}

\section{SEAN M. HAMMOND \\ University of Surrey, Guildford, Surrey, England}

Factor indeterminacy has been recognized as a problem for factor analysts since the early developments of the method (Steiger \& Schonemann, 1978). Briefly stated, indeterminacy refers to the finding that for any common factor solution, there exist more than one set of legitimate factor scores. More generally, it may be shown (Maxwell, 1971, 1972) that indeterminacy may be conceptualized as the correlation between the "true" and the "estimated" factors. Indeterminacy, then, may be seen as a problem of factor reliability.

Despite claims that the existence of indeterminacy is sufficiently problematic to throw doubt on the utility of the common factor model (Guttman, 1955; Schonemann \& Wang, 1972), very little dissemination of the problem appears to have taken place among substantive users of factor analysis. This apparent ignorance probably stems from three sources. First, the problem is statistically complex and has been debated mainly in a highly technical manner (Elffers, Bethlehem, \& Gill, 1978; McDonald, 1977; Schonemann, 1971), which may be poorly understood by mathematically naive researchers. Two notable exceptions to this rule are the papers by McDonald and Muliak (1979) and Steiger and Schonemann (1973).

Second, there is still considerable controversy as to the seriousness of indeterminacy in practical factor analysis. Gorsuch (1974) expressed the opinion that indeterminacy only becomes a serious problem when factor scores are required, and that it is of negligible concern in analyses where interest is centered upon identifying factors as constructs. Guttman (1957), on the other hand, argued that attaching meaning to factors on the basis of loadings is highly suspect where indeterminacy exists. Steiger and Schonemann (1978) concluded their review of the problem by stating that, whomever you believe, the fact of indeterminacy means that factor analysts have been operating at a suboptimal level.

Finally, apparent lack of interest in the indeterminacy problem probably is due in part to the increasing automation that computers have brought to factor analysis, in which the arrival at a factor solution takes clear precedence over the assessment of the appropriateness of the common factor model to the data in question. Despite the fact that a number of quite straightforward procedures exist for the assessment of factor indeterminacy (Budescu, 1983; Green, 1976; Guttman, 1955; Maxwell, 1972), there are very few accessible computer routines for the

The author's mailing address is: Department of Psychology, University of Surrey, Guildford, Surrey, GU2 5XH, England. analyst to use. With the availability of programs for generating canonical or maximum likelihood factor solutions, these techniques have become all the more feasible.

The purpose of this paper is to report on a portable program, DETFAC, for estimating the indeterminacy of each factor from a given factor loading matrix. McDonald (1974) suggested that the squared multiple correlation of the factor score regression equation, $\varrho^{2}$, is the most applicable measure of factor indeterminacy. This value may be seen as the expected correlation between pairs of arbitrary factor solutions (Muliak, 1976). Some authors (Green, 1976; Maxwell, 1972) have supported claims for the utility of $\varrho^{2}$ in assessing indeterminacy, whereas others (Elffers et al., 1978; Schonemann, 1971; Schonemann \& Wang, 1972) have argued for the more stringent criterion proposed by Guttman (1955). These two measures, although conceptually quite distinct, are related by the formula

$$
\varrho^{2}=(\gamma+1) / 2,
$$

where $\gamma$ represents Guttman's criterion. DETFAC calculates both of these measures.

The Formulas. The algorithm used by DETFAC is quite simple. Given the $p \times n$ factor loading matrix $\Lambda$, where $p$ is the number of variables and $n$ is the number of factors, it is relatively trivial to calculate the $n \times n$ matrix $\Delta$ :

$$
\Delta=\Lambda^{\prime} \Psi^{-1} \Lambda,
$$

where $\Psi$ is a $p \times p$ diagonal matrix of variable uniquenesses.

The squared correlations between the true and estimated factors may be derived from the diagonal of the following matrix:

$$
\Delta\left[(\mathrm{I}+\Delta)^{-1}\right],
$$

which may be reduced for computational ease to

$$
\varrho_{i}^{2}=\delta_{i i} /\left(1+\delta_{i i}\right) \quad(i=1 \ldots n) .
$$

It may be clearly seen that $\varrho_{i}^{2}$ is Maxwell's (1971) reliability coefficient for the $i$ th factor.

In order to calculate Guttman's (1955) criterion value for each factor, the following matrix formula (from Elffers et al., 1978) may be used:

$$
\text { DIAG }\left[\mathrm{I}-2(\mathrm{I}+\Delta)^{-1}\right] \text {. }
$$

Thus,

$$
\gamma_{i}=1-2 /\left(\delta_{i i}+1\right) \quad(i=1 \ldots n) .
$$

DETFAC also will calculate the Guttman criterion for rotated factors. In this case the program requires the additional input of either the $n \times n$ transformation matrix, T, for orthogonal rotations or the $n \times n$ interfactor correlation matrix, $\theta$, for oblique factors. 
For orthogonal rotations it can be shown (Elffers et al., 1978) that the diagonal of the following product matrix will contain the indeterminacy coefficients for each factor:

$$
\mathrm{T}^{\prime}\left[\mathrm{I}-2(\mathrm{I}+\Delta)^{-1} \mathrm{~T}\right] \text {. }
$$

For oblique rotations the coefficients are obtained by utilizing the following formula:

$$
\operatorname{DIAG}\left[\theta-2\left(\theta^{-1}+\Delta\right)^{-1}\right]
$$

reducing to:

$$
\gamma_{i}=1-2 /\left(\tilde{\theta}_{i i}+\delta_{i i}\right) \quad(i=1 \ldots n),
$$

where $\tilde{\theta}_{i i}$ is a diagonal element of $\Theta^{-1}$.

The Program. DETFAC is written in standard FORTRAN 77 and has no special compiling requirements. It has been used successfully on an IBM 370 and on a PRIME 750 and is intended to be fully portable.

Input. DETFAC requires the $p \times n$ unrotated factor matrix to be available on an external file with the factors in columns and the variables in rows. If the solution has been rotated orthogonally, the transformation matrix is expected on the same file and in the same format immediately following the factor matrix. For oblique rotations the factor pattern matrix and the interfactor correlation matrix are required. The program will interactively request the name of the file on which the data is stored, the number of variables $(p)$, the number of factors $(n)$, and the row format of the data matrices in $F$ notation, as well as any rotation specifics.

Output. A table of $\gamma$ and $\varrho^{2}$ values is output for each factor entered into the program.

Availability. Documentation and a listing of DETFAC are available free of charge from the author.

\section{REFERENCES}

Budescu, D. V. (1983). The estimation of factor indeterminacy. Educational \& Psychological Measurement, 43, 971-976.

ElfFers, H., Bethlehem, J., \& Gill, R. (1978). Indeterminacy problems and the interpretation of factor analysis results. Statistica Neerlandica, 33, 185-199.

GorSuCH, R. L. (1974). Factor analysis. Philadelphia: Saunders

GreEn, B. F. (1976). On the factor score controversy. Psychometrika, 41, 263-266.

GutTman, L. (1955). The determinacy of factor score matrices with implications for five other basic problems of common factor theory. British Journal of Statistical Psychology, 8, 65-81.

GutTman, L. (1957). Simple proofs of relations between the communality problem and multiple correlation. Psychometrika, 22, 147-157.

MAXwELL, A. E. (1971). Estimating true scores and their reliabilities in the case of composite psychological tests. British Journal of Mathematical \& Statistical Psychology, 24, 195-204.

MAXWELL, A. E. (1972). Factor analysis: Thompson's sampling theory recalled. British Joumal of Mathematical \& Statistical Psychology, 25, 1-21.

MCDonald, R. P. (1974). The measurement of factorial indeterminacy. Psychometrika, 39, 203-221.

MCDonalD, R. P. (1977). The indeterminacy of components and the definition of common factors. British Journal of Mathematical \& Statistical Psychology, 30, 165-176.

McDonald, R. P., \& Muliak, S. A. (1979). Determinacy of common factors: A nontechnical review. Psychological Bulletin, 86, 297-306.

Muliak, S. A. (1976). Comments on "The measurement of factorial indeterminacy." Psychometrika, 41, 249-262.

SCHONEMANN, P. H. (1971). The minimum average correlation between equivalent sets of uncorrelated factors. Psychometrika, 36, 21-30.

SchonemanN, P. H., \& WaNG, M. M. (1972). Some new results on factor indeterminacy. Psychometrika, 37, 61-91.

Steiger, J. H., \& SchonemanN, P. H. (1978). A history of factor indeterminacy. In S. Shye (Ed.), Theory construction and data analysis in the behavioral sciences (pp. 136-178). London: Jossey-Bass.

(Revision accepted for publication April 16, 1987.)

\title{
NOTICE
}

\author{
Special Issue \\ Psychobiology \\ Call for Manuscripts
}

A special issue of Psychobiology is being planned in the area of Psychobiology of Sexual Differentiation and Gender-Related Behaviors. It will include both empirical research and review papers. Manuscripts should review empirical literatures, advance theory or present major sets of data. Publication of the special issue is planned for Fall 1988. The deadline for initial submissions is March 1, 1988.

Contributions intended for the special issue should be sent to the guest editor: Jane Stewart, Center for Studies in Behavioral Neurobiology, Concordia University, 1455 de Maisonneuve Blvd. West, Montreal, PQ H3G 1M8, Canada. 